\title{
Tissue engineering in cardiovascular surgery: MTT, a rapid and reliable quantitative method to assess the optimal human cell seeding on polymeric meshes ${ }^{1}$
}

\author{
Gregor Zund*, Qing Ye, Simon P. Hoerstrup, Andreina Schoeberlein, \\ Andreas C. Schmid, Jurg Grunenfelder, Paul Vogt, Marko Turina \\ Clinic for Cardiovascular Surgery, University Hospital Zurich, Zurich, Switzerland
}

Received 22 September 1998; received in revised form 27 January 1999; accepted 2 February 1999

\begin{abstract}
Objective: Currently used valve substitutes for valve replacement have certain disadvantages that limit their long-term benefits such as poor durability, risks of infection, thromboebolism or rejection. A tissue engineered autologous valve composed of living tissue is expected to overcome these shortcomings with natural existing biological mechanisms for growth, repair, remodeling and development. The aim of the study was to improve cell seeding methods for developing tissue-engineered valve tissue. Methods: Human aortic myofibroblasts were seeded on polyglycolic acid (PGA) meshes. Cell attachment and growth of myofibroblasts on the PGA scaffolds with different seeding intervals were compared to determine an optimal seeding interval. In addition, scanning electron microscopy study of the seeded meshes was also performed to document tissue development. Results: There was a direct correlation between cell numbers assessed by direct counting and MTT(3-[4,5-dimethylthiazol-2-yl]-2,5-diphenyltertra-zolium bromide) assay. Both attach rate and cell growth seeded on meshes with long intervals ( 24 and $36 \mathrm{~h})$ were significantly higher than those seeded with short intervals $(2$ and $12 \mathrm{~h})(P<0.01)$, there was no significant difference between 24- and 36-h seeding interval. Scanning electron microscopy also documented more cell attachment with long seeding intervals resulting in a more solid tissue like structure. Conclusion: It is feasible to use human aortic myofibroblasts to develop a new functional tissue in vitro. Twenty-four hours is an optimal seeding interval for seeding human aortic myofibroblasts on PGA scaffolds and MTT test is a rapid and reliable quantitative method to assess the optimal human cell seeding on polymeric meshes. (C) 1999 Elsevier Science B.V. All rights reserved.
\end{abstract}

Keywords: Tissue engineering; Heart valves; Cell seeding; Seeding intervals; MTT

\section{Introduction}

Valvular heart disease is a significant cause of morbidity and mortality world wide. The state-of-the-art prosthetic valves clinically used include xenografts, mechanical valve and homografts. They all have certain limitations such as poor durability, foreign body reaction, infection, anticogulation problem or donor scarcity.

The limitations associated with various valve substitutes have motivated the exploration of novel valve substitutes. A

\footnotetext{
* Corresponding author. Tel.: +41-1-255-1111; fax: +41-1-255-4369; e-mail: gregor.zund@chi.usz.ch

${ }^{1}$ Presented at the 12th Annual Meeting of the European Association for Cardio-thoracic Surgery, Brussels, Belgium, September 20-23, 1998.
}

series of studies have been undertaken to determine if tissue engineering principles could be used to develop valve tissue substitutes. Tissue engineering is a multidisciplinary science that uses basic principles from engineering and biology to construct tissue from their cellular components $[1,2]$. The advantage of this approach over whole organ/tissue transplantation is that only the cells of interest are implanted, and they can be potentially multiplied in vitro. The use of human autologous cells and/or extracellular matrix further eliminates the rejection and donor scarcity.

In cardiovascular surgery, using autologous cells to create an autologous tissue engineered heart valve will offer several theoretical advantages over the presently used valve substitutes. An autologous bioprosthetic valve will be a living structure, and therefore, it should be able to demonstrate 
the natural existing biological mechanisms for repair and remodeling. Theoretically, a greater durability can be expected [3]. A tissue-engineered autologous heart valve will be completely biocompatible, with minimal risk of infection and thromboemlolic complications and without risk of rejection. There might even be a potential for further growth, which might be important for the pediatric patients. The group at Boston Children's Hospital had successfully created tissue-engineered heart valve leaflets with animal cells and implanted these leaflets at the pulmonary leaflet position in lamb models. The leaflets functioned well in the pulmonary circulation of growing lambs for up to 4 months and no stenosis nor regurgitation could be seen [4,5].

With the obtained knowledge from animal cell study, we therefore decided to create a tissue-engineered human heart valve. We first focused upon the development and evaluation of an adequate method. For this reason we started with creating a living compound of human skin fibroblasts followed by seeding of human aortic endothelial cells. The results showed that the human skin fibroblast after 3 weeks were attached to the polymeric fibers and had spread out and divided. The seeded endothelial cells formed a monolayer over the human fibroblasts and no invasive growth of endothelial cells nor a new formation of capillaries could be detected. The idea of creating a heart valve in vitro was not only working with animal cells but with human cells [6].

Seeding of human fibroblasts on biodegradable mesh is essential for the creation of human autologous tissue-engineered valve. Efforts have been made to increase cell attachment to polymer scaffolds for the purpose of tissue engineering. Improved cell attachment are achieved with dynamic (stirred seeding or agitated seeding technique) seeding [7,8], or precoating polymer scaffold with adhesion factors [9].

We had used several different seeding intervals in previous studies, but which seeding interval might be optimal for rapid cell attachment and enhanced growth remained unclear. In our study, we focus on the seeding interval to improve cell seeding, a rapid colorimetric assay, MTT (3[4,5-dimethylthiazol-2-yl]-2,5-diphenyltertra-zolium bromide) test was employed to decide an optimal cell seeding interval for seeding human aortic myofibroblast cells on to polyglycolic acid (PGA) polymer scaffolds.

\section{Materials and methods}

\subsection{Polymer scaffolds}

Non-woven PGA polymer (a kindly gift of Dr. Vacanti, Children's Hospital, Harvard Medical School, Boston, MA) was used as three-dimensional scaffolds. The fiber diameter is $12-15 \mu \mathrm{m}$ with a polymer density of $70 \mathrm{mg} / \mathrm{ml}$. The resorption capacity was $4-6$ weeks by hydrolysis. In the present study, meshes were used as square sheets of
$1.0 \times 1.0 \times 0.3 \mathrm{~cm}$ in size, cold gas (ethylene oxide) sterilized and were rinsed with phosphate buffered saline (PBS) before cell seeding to remove the toxic gas residues.

\subsection{Human aortic myofibroblast cell expansion}

Human aortic tissue was harvested from the ascending aorta of donor hearts for heart transplantation. After harvesting, the explants were rinsed off blood with PBS and stripped off adventitia with scissors under a laminar flow hood (Forma Scientific, OH). Thereafter, the explants were digested with collagenase (Collagenase Type I, Worthington Biochemical Corporation, Freehold, NJ, prepared as a solution of $75-80 \mu / \mathrm{ml}$ in PBS) for $30 \mathrm{~min}$ at room temperature. After digestion, the endothelial cells were detached using a rubber cell scraper and the explants were then cut into small pieces of $2 \times 2 \mathrm{~mm}$. The explants were evenly distributed over the bottom of $75 \mathrm{~cm}^{2}$ vented polystyrene cell culture flasks (Falcon 3111, Becton Dickinson, Lincoln Park, NJ). After the explants were firmly attached to the bottom of the flasks, Dulbecco's Modified Eagles Medium supplemented with $10 \%$ fetal bovine serum and $1 \%$ streptomycin solution (Gibco BRL-Life Technologies, Grand Island, NY) were gently added, taking care not to dislodge the tissue pieces. The explants were placed in a humidified incubator (Forma Scientific, $\mathrm{OH}$ ) at $37^{\circ} \mathrm{C}$ with $5 \% \mathrm{CO}_{2} / 95 \%$ air. After 5-7 days of culture, cells began to migrate off the tissue pieces and onto the flask bottoms to form mixed cell populations of fibroblasts and smooth muscle cells (myofibroblast). After 3-4 weeks, the mixed cell populations grew into confluent monolayers, they were serially passaged by trypsinization (trypsin/EDTA solution, 0.05/0.02\%, Gibco BRL-Life Technologies, 3-5 min) and subcultured to obtain sufficient cell numbers for polymer scaffold seeding (passage 3-4). Medium was changed twice a week.

\subsection{Cell seeding}

Human aortic myofibroblasts were trypsinized from culture flasks and suspended to create a single cell suspension. Cell number was determined by direct counting with Neubauer counting chamber (Brand, Germany). The cell suspension was centrifuged at $1000 \mathrm{rpm}$ at $20^{\circ} \mathrm{C}$ for 5 min, the supernatant was sucked off and fresh culture medium was added to create a new single cell suspension with desired seeding cell number per $100 \mu \mathrm{l}$ volume. Seedings were performed in polystyrene 6-well flat-bottom culture plate (Costar 3516, Cambridge, MA), PGA scaffolds were placed in the center of the wells, $100 \mu \mathrm{l}$ cell suspension of desired seeding number was evenly distributed onto each polymer mesh. After $2 \mathrm{~h}$ culture in incubator, allowing full attachment of cells to scaffolds, medium was added $5 \mathrm{ml} /$ well for further culture. The medium had to be sucked off before each seeding. In addition, medium was changed daily. 


\subsection{MTT assay}

MTT assay determines viable cell numbers and is based on the mitochondrial conversion of the tetrazolium salt, 3-[4,5-dimethylthiazol-2-yl]-2,5-diphenyltertrazolium bromide (MTT). Modified MTT assay was employed in this study to quantitatively assess the viable cell numbers of human aortic myofibroblasts attached and grew on PGA scaffolds. Briefly, $500 \mu \mathrm{l}$ serum free medium and $40 \mu \mathrm{l}$ MTT solution $\left(5 \mathrm{mg} / \mathrm{ml}\right.$ in $\mathrm{Ca}^{2+}$ and $\mathrm{Mg}^{2+}$ free PBS, Sigma, St. Louis, MO) were added to each sample, and incubated at $37^{\circ} \mathrm{C}$ for $1 \mathrm{~h}$ for MTT formazan formation. The medium and MTT were replaced by isopropanol solution (containing $10 \%$ formic acid; Sigma, St. Louis, MO), $400 \mu$ l per well, the samples were incubated at $37^{\circ} \mathrm{C}$ for additional $5 \mathrm{~min}$, to solve the MTT formazan, also mildly shaken for $10 \mathrm{~min}$ to ensure the dissolution of formazan. MTT dissolved formazan solution (100 $\mu \mathrm{l}$ ) was added into 96-well $\mu \mathrm{l}$ plates (Falcon 3072, Becton Dickinson, Lincoln Park, NJ) per well and the absorbency values was measured using an ELISA reader (Dynatech 5000, Dynatech, Billinghurst) at wavelength 550 $\mathrm{nm}$, blanked with isopropanol solution. Two replicates were read for each sample, the mean value of the two was used as the final result. MTT was first performed on a directly counted human myofibroblast cell serial $\left(0.5 \times 10^{5}, 1.0 \times 10^{5}, 1.5 \times 10^{5}, 2.0 \times 10^{5}, 2.5 \times 10^{5} \mathrm{ml}\right)$, the absorbency values were plotted against the counted cell numbers to establish a standard calibration curve. Viable cell numbers on meshes or culture well bottom were then determined from the standard curve according to their MTT absorbency.

\subsection{Cell attachment and seeding intervals}

Human aortic mixed cell populations (myofibroblasts, $0.5 \times 10^{6}$ ) were seeded onto PGA meshes consecutively for six times with four different intervals: 2-, 12-, 24- and 36-h. Cultured for 2 weeks after first seeding, the cell numbers attached and grew on the PGA meshes $(n=4)$ as well as the cell numbers unattached and grew on the culture plate bottom $(n=6)$ were determined by MTT assay. The cell numbers both grew on PGA meshes and in culture wells were compared among different seeding interval groups to determine an optimal seeding interval, from which a higher viable cell number on PGA meshes but a lower cell number in culture well were expected. Electron microscopy scanning was also performed on meshes from each group to evaluate tissue development.

\subsection{Scanning electron microscopy (SEM)}

Samples for SEM examination were fixed in $2 \%$ glutaraldehyde solution, and gradient dehydrated at critical point followed by AuPd sputtering. Samples were observed in JSM-255 scanning microscope (JEOL, Japan).

\subsection{Statistics}

Results data were expressed as mean \pm standard error of the mean (SEM). Comparisons between groups were performed by ANOVA test. Statistical significance was set at $P<0.05$. Linear regression analysis was utilized to evaluate the correlation. Data and graphs were proceeded with StatView 4.5 (Abacus Concepts, Berkeley, CA).

\section{Results}

\subsection{Correlation between MTT assay and direct cell counting}

MTT was performed on directly counted human aortic myofibroblast serial. Linear regression analysis of the results showed that there was a linear correlation between the cell numbers directly counted and the MTT absorbency at $550 \mathrm{~nm}, y=-0.056+2.632 \times 10^{-6} x, r^{2}=0.994$ (Fig. 1). The presence of viable cell numbers on PGA scaffolds in the following experiments were determined by means of the calibration curve.

\subsection{Optimal seeding interval}

The same numbers of human myofibroblasts were seeded onto the non-woven PGA meshes with four different seeding intervals, namely 2-, 12-, 24-, 36-h. The myofibroblasts attached to the PGA meshes, proliferated and produced extracellular matrix on the meshes and grew into new tissue-like architectures. After 2 weeks of culture after first seeding, MTT assay was performed on these meshes, absorbency values were recorded, and the cell numbers on the meshes were determined from the standard (Table 1). After

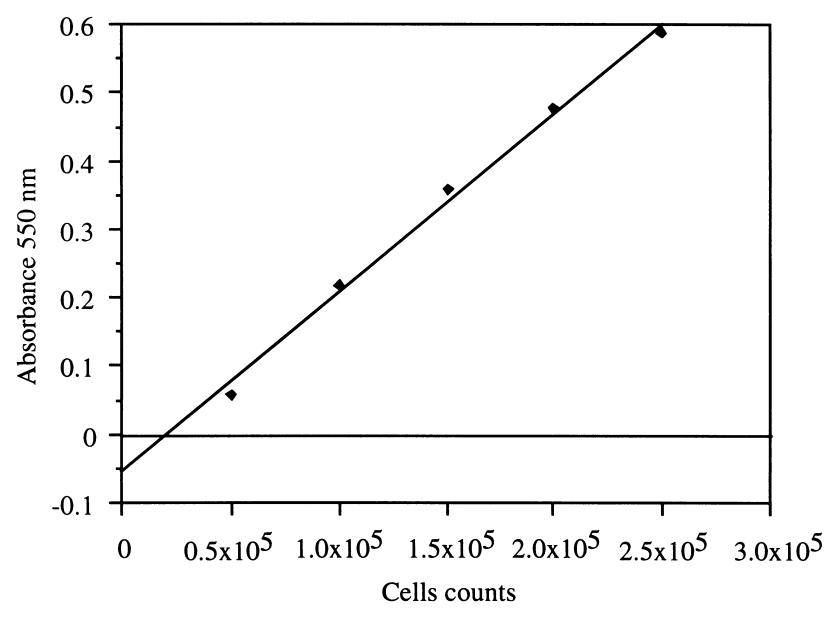

Fig. 1. MTT absorbency values at $550 \mathrm{~nm}$ were recorded and plotted against their counterparts in directly counted human aortic myofibroblast cell serial. There was a linear correlation between the cell numbers directly counted and the MTT absorbency, $y=-0.056+2.632 \times 10^{-6} x$, $r^{2}=0.994$. 
Table 1

MTT absorbency at $550 \mathrm{~nm}$ of cells on PGA meshes and viable cell numbers determined from standard curve in different seeding interval groups $(n=4)$

\begin{tabular}{lllr}
\hline Seeding intervals $(\mathrm{h})$ & 2 & 12 & 24 \\
\hline OD MTT & $0.697 \pm 0.033$ & $1.012 \pm 0.070$ & $1.260 \pm 0.026$ \\
Cell number $\left(\times 10^{6}\right)$ & $0.285 \pm 0.013$ & $0.404 \pm 0.026$ & $0.498 \pm 0.097$ \\
\hline
\end{tabular}

each seeding, the unattached myofibroblasts fished out from the meshes to the culture wells and grew on the bottoms of the culture wells. MTT was also performed on these cells (Table 2). The results showed that the cell numbers unattached to the PGA meshes and growing on the bottoms of the culture wells were significantly higher in 2- and 12-h seeding interval groups compared with those in 24- and 36-h seeding interval groups $(P<0.01)$, while the cell numbers attached and growing on the PGA meshes were significantly lower in the 2- and 12-h seeding interval groups compared with those in 24- and 36-h seeding interval groups $(P<0.01)$. There was no significant difference of viable cell numbers of both unattached and attached cells between the two long seeding interval groups (24- and 36-h, $P>0.05)$, while there was significant difference between the 2 - and 12-h seeding groups $(P<0.01)$, as shown in Fig. 2.

\subsection{Scanning electron microscopy}

The meshes seeded with long intervals formed more solid tissue sheets. Electron microscope scanning examinations revealed the attachment of the fibroblasts to the polymer fibers and a distribution of myofibroblasts throughout the polymeric architecture in each seeding group. Compared with the meshes seeded with short intervals (2- and 12-h), the cells on PGA meshes seeded with long intervals (24-, 36-h), formed a much more confluent continuous myofibroblasts-polymer architecture (Fig. 3). And there was no obvious difference on meshes seeded with 24- and 36-h intervals, which were coherent with the results of viable cell numbers assessed by MTT assay.

The results suggested that longer seeding intervals (24- or 36-h) were optimal for cell seeding which resulted in a higher cell attachment and growth on the PGA scaffolds than short seeding intervals did, and there was no significant difference of cell attachment and growth between two longer seeding intervals (24- and 36-h).

\section{Discussion}

In our previous study, we successfully used human foreskin fibroblasts and human aortic endothelial cells to develop a valve tissue in vitro. The cellular component of normal heart valve tissue is composed mainly of endothelial cells and myofibroblasts. Therefore, tissue for cell harvesting and in vitro expanding can be obtained from vascular structures that contain similar cell components [3]. Based on this consideration, we used human aortic myofibroblast in the present study.

In cardiovascular tissue engineering three-dimensional polymer scaffold is used to mimic its natural counterparts, the extracellular matrices of the body [10]. It serves as both a physical support and as adhesive substrate for seeded cells during in vitro culture and subsequent implantation. The cells must first attach to three-dimensional biodegradable polymer substrate then spread out before they start proliferating and developing into a tissue-like structure. Furthermore, the cell-polymer configuration allows for gas exchange and diffusion of nutrients [6]. As the transplanted cells grow and function normally, they begin to secrete their own ECM support. Concurrently, the scaffold degrades and is eliminated as the need for an artificial support diminishes.

Cell distribution and attachment in three dimensions are mainly hampered by the force of gravity, therefor, rapid attachment to the polymer surface is important [11]. Cell adhesion to both natural and synthetic substrates is mediated by cell attachment and spreading factors presented in the extracellular matrix in vivo [12]. These attachment and spreading factors are normally cellular in origin, trypsinized cells, not surprisingly, often lack the capacity of adhere following subcultures. When trypsinized cells are seeded, they enter a lag period of 2-24 h, followed by a period of exponential growth and finally enter a period of reduced or zero growth after they become confluent [13]. Given no extrogenous adhesion factors, the previous existing cells on substrate might play an important role for the attachment

Table 2

MTT absorbency at $550 \mathrm{~nm}$ of cells on culture well bottom and viable cell numbers determined from standard curve in different seeding interval groups $(n=6)$

\begin{tabular}{lllr}
\hline Seeding intervals $(\mathrm{h})$ & 2 & 12 & 24 \\
\hline OD MTT & $1.529 \pm 0.035$ & $0.986 \pm 0.032$ & $0.407 \pm 0.019$ \\
Cell number $\left(\times 10^{6}\right)$ & $0.600 \pm 0.013$ & $0.394 \pm 0.012$ & $0.440 \pm 0.016$ \\
\hline
\end{tabular}




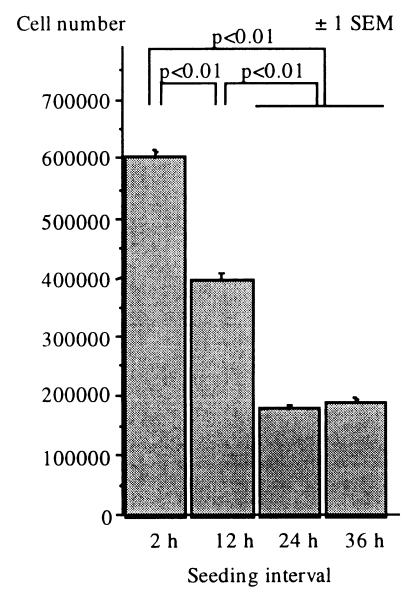

B

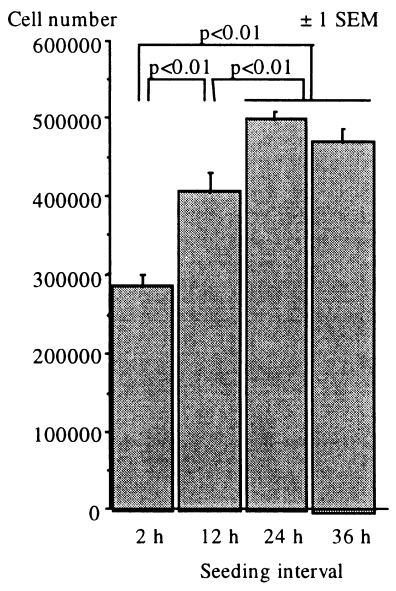

Fig. 2. Comparison of viable numbers on the culture well bottom (A, $n=6)$ and the cells on PGA scaffolds (B, $n=4)$ among the four different seeding interval groups. Data represent the mean \pm SEM.

of subsequently seeded cells to the substrate, through providing attachment and spreading factors to enhance cellpolymer and cell-cell attachment. In the presented study, we find a significant higher cell attachment and growth on PGA meshes seeded with long intervals (24- and 36-h) compared with that on meshes seeded with short intervals (2and $12-\mathrm{h})$. This could potentially be explained by the 'preconditioning' of the meshes by the previous seeded cells. In the long seeding interval groups, the previous seeded cells enter the period of exponential growth and begin to spread, proliferate and secret, thus provide more optimal environment for cell attachment of the following seedings. There is no difference in cell attachment and growth between the two long seeding intervals. Compared with $36-\mathrm{h}$ seeding interval, 24-h seeding is more convenient to be performed, we decided this as an optimal seeding interval for the current purpose of study.

MTT assay was originally described by Mosmann [14]as a useful method for the measurement of in vitro cytotoxicity and cell proliferation. It is a semi-automated colorometric assay which determines viable cell numbers and is based on the conversion by mitochondrial succinate dehydrogenases of the tetrazolium salt, 3-[4,5-dimethylthiazol-2-yl]-2,5diphenyltertra-zolium bromide (MTT), a water-soluble yellow dye, to a water-insoluble purple formazan product that has been shown to be proportional to the numbers of living cells presented. The formazan is then dissolved in isopropanol and the absorbency is recorded in photometer. The tetrazolium ring is cleaved in active mitochondria, so the reaction occurs only in living cells. The MTT assay has been demonstrated as a sensitive, precise, convenient, rapid and economical test method by many studies[15-17]. Furthermore, the advantages of this colorimetric assay is the lack of any radioisotope. This study also proves positive correlation among direct cell counting, MTT assay and ${ }^{3} \mathrm{H}$-thymidine incorporation and DNA measurement in three-dimensional human peritoneal fibroblast culture [18]. Our result demon-

strates a linear correlation between directly counted human aortic myofibroblast cell number and MTT absorbency.

In conclusion, this study demonstrates the feasibility of using human ascending aortic myofibroblasts seeded on biodegradable polymer scaffold to develop a new functional tissue in vitro with application of tissue engineering principles. Seeding techniques is essential for rapid cell attachment, spreading and proliferation on polymer scaffolds for in vitro culture. MTT test is a rapid and reliable quantitative method to assess the optimal human cell seeding on polymeric meshes and 24-h is an optimal seeding interval for seeding human aortic myofibroblasts on PGA scaffolds.

These preliminary results are promising, and represent a basic step toward construction of human autologous cardiovascular valve tissue. However, the engineering of autologous cardiovascular structure is still in an early stage of development, and numerous issues remain to be investigated.
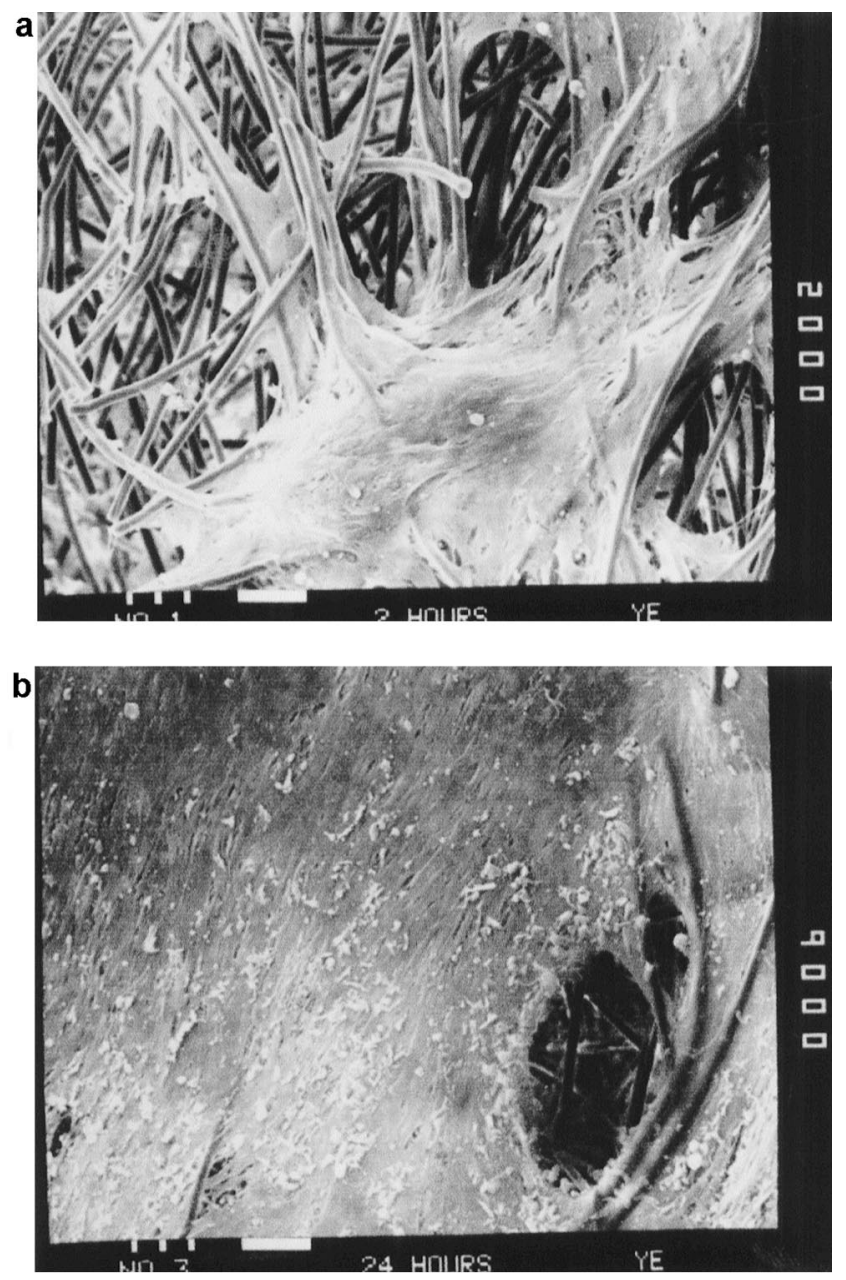

Fig. 3. Scanning electron microscopy documented the attachment of myofibroblasts to the polymer fibers and a distribution of myofibroblasts throughout the PGA meshes. Compared with the mesh seeded with 2-h seeding interval (A), the mesh seeded with 24-h seeding interval (B) formed a much more confluent continuous myofibroblast-polymer architecture. 


\section{References}

[1] Langer R, Vancanti JP. Tissue engineering. Science 1993;260:920960.

[2] Vancanti JP. Beyond transplantation. Third annual Samuel Jason Mister lecture. Arch Surg 1988;123:545-549.

[3] Shinoka T, Mayer JE. New frontiers in tissue engineering heart valves. In: Atala A, Mooney D, editors. Synthetic biodegradable polymer scaffolds. Boston: Birkhäuser, 1997:187-198.

[4] Shinoka T, Breuer CK, Tanel RE, Zund G, Miura T, Ma PX, Langer R, Vacanti JP, Mayer JE. Tissue engineering heart valves: valve leaflet replacement study in a lamb model. Ann Thorac Surg 1995;60:S513-S516.

[5] Shinoka T, Ma PX, Shum-Tim D, Breuer CK, Cusick RA, Zund G, Langer R. Tissue-engineered heart valves: autologous valve leaflet replacement in a lamb model. Circulation 1996;94 (Suppl. II):II164II168.

[6] Zund G, Hoerstrup SP, Shoeberlein A, Lachat M, Ulschmid G, Vogt $\mathrm{P}$, Turina $\mathrm{M}$. Tissue engineering: a new approach in cardiovascular surgery; seeding of human fibroblasts followed by human endothelial cells on resorbable mesh. Eur J Cardio-thorac Surg 1998;13:160 164.

[7] Kim BS, Putnam AJ, Kulik TJ, Mooney DJ. Optimizing seeding and culture methods to engineer smooth muscle tissue on biodegradable polymer matrices. Biotechnol Bioeng 1998;57:46-54.

[8] Vunjak-Novakovic G, Obradovic B, Martin I, Bursac PM, Langer R, Freed LE. Dynamic cell seeding of polymer scaffolds for cartilage tissue engineering. Biotechnol Prog 1998;14:193-202.

[9] Sittinger M, Schultz O, Keyszer G, Minuth WW, Burmester GR. Artificial tissue in perfusion culture. Int J Artif Organs 1997; 20:57-62.
[10] Vacanti CA, Vacanti JP. Bone and cartilage reconstruction with tissue engineering approaches. Otolaryngol Clin North Am 1994; 27:263-276.

[11] Sittinger M, Bujia J, Rotter N, Reituel D, Minuth WW, Burmester GR. Tissue engineering and autologous transplant formation: practical approaches with resorbable biomaterials and new cell culture techniques. Biomaterials 1996;17:237-242.

[12] Massia SP, Hubbell JA. Tissue engineering in the vascular graft. Cytotechnology 1992;10:189-204.

[13] Freshney RI. Animal cell culture: a practical approach. 2nd edn. Oxford: IRL Press, 1992:11.

[14] Mosmann T. Rapid colorimetric assay for cellular growth and survival: application to proliferation and cytotoxicity assays. J Immunol Methods 1983;65:55-63.

[15] Hongo T, Fujii Y, Igarashi Y. An in vitro chemosensitivity test for the screening of anti-cancer drugs in childhood leukemia. Cancer 1990;65:1263-1272.

[16] Wan H, Williams R, Doherty P, Williams DF. A study of the reproducibility of the MTT test. J Mater Sci: Mater in Med 1994;5:154159.

[17] Heo DS, Park JG, Hata K, Day R, Herberman RB, Whiteside TL. Evaluation of tetrazolium-based semiautomatic colorimetric assay for measurement of human antitumor cytotoxicity. Cancer Res 1990;50:3681-3690.

[18] Beavis MJ, Willians JD, Hoppe J, Topley N. Human peritoneal fibroblast proliferation in 3-dimensional culture: modulation by cytokines, growth factors and peritoneal dialysis effluent. Kidney Int 1997;51:205-215. 